\title{
The CXCR4-CXCL12 axis in Ewing sarcoma: promotion of tumor growth rather than metastatic disease
}

Dagmar Berghuis ${ }^{1,2}$, Marco W Schilham², Susy J Santos ${ }^{2}$, Suvi Savola ${ }^{3}$, Helen J Knowles ${ }^{4}$, Uta Dirksen ${ }^{5}$, Karl-Ludwig Schaefer ${ }^{6}$, Jukka Vakkila ${ }^{3}$, Pancras CW Hogendoorn ${ }^{1}$ and Arjan C Lankester ${ }^{2^{*}}$

\begin{abstract}
Background: Chemokine receptor CXCR4, together with its ligand CXCL12, plays critical roles in cancer progression, including growth, metastasis and angiogenesis. Ewing sarcoma is a sarcoma with poor prognosis despite current therapies, particularly for patients with advanced-stage disease. Lungs and bone (marrow), organs of predilection for (primary/metastatic) Ewing sarcoma, represent predominant CXCL12 sources.

Methods: To gain insight into the role of the CXCR4-CXCL12 axis in Ewing sarcoma, CXCR4, CXCL12 and hypoxia-inducible factor-1 a protein expression was studied in therapy-naive and metastatic tumors by immunohistochemistry. CXCR4 function was assessed in vitro, by flow cytometry and proliferation/ cell viability assays, in the presence of recombinant CXCL12 and/or CXCR4-antagonist AMD3100 or under hypoxic conditions.

Results: Whereas CXCR4 was predominantly expressed by tumor cells, CXCL12 was observed in both tumor and stromal areas. Survival analysis revealed an (expression level-dependent) negative impact of CXCR4 expression $(p<0.04)$. A role for the CXCR4-CXCL12 axis in Ewing sarcoma growth was suggested by our observations that i) CXCR4 expression correlated positively with tumor volume at diagnosis $(p=0.013)$, ii) CXCL12 was present within the microenvironment of virtually all cases, iii) CXCL12 induced proliferation of CXCR4-positive Ewing sarcoma cell lines, which could be abrogated by AMD3100. CXCR4 expression was not correlated with occurrence of metastatic disease. Also, therapy-naïve tumors demonstrated higher CXCR4 expression as compared to metastases ( $p=0.027$ ). Evaluation of in vivo hypoxia-inducible factor-1a expression and culture of cells under hypoxic conditions revealed no role for hypoxia in CXCR4 expression.
\end{abstract}

Conclusions: Together, our results imply a crucial role for the CXCR4-CXCL12 axis in auto- and/or paracrine growth stimulation. Integration of CXCR4-targeting strategies into first- and/or second-line treatment regimens may represent a promising treatment option for Ewing sarcoma.

Keywords: Ewing sarcoma, CXCR4, CXCL12 (stromal-cell derived factor-1 (SDF-1)), Chemokine, Growth signaling, Hypoxia, Metastasis, Prognosis, Therapy

\footnotetext{
* Correspondence: a.lankester@lumc.nl

${ }^{2}$ Department of Pediatrics, Leiden University Medical Center, Albinusdreef 2, 2300, RC Leiden, the Netherlands

Full list of author information is available at the end of the article
} 


\section{Background}

The chemokine network, initially described as an essential mediator of directional cell migration in inflammation and immune cell homing, has become increasingly recognized as contributing to a broad spectrum of other physiological and pathological processes, including cancer [1]. Although cancers of different histological origin express different chemokine receptors and/or (corresponding) ligands, chemokine receptor CXCR4 together with its cognate ligand CXCL12 (stromal cell-derived factor-1/SDF-1) is the most widely expressed (as reviewed by [2]). Constitutive CXCR4 expression has been detected in a range of adult tissues, including hematopoietic cells, vascular smooth muscle and endothelial cells and epithelial cells of different origin, whereas CXCL12 is constitutively expressed by stromal cells within the lungs and bone marrow microenvironment [2]. Hypoxia-inducible factor- $1 \alpha$ (HIF-1 $\alpha$ ), a wellcharacterized inducer of gene transcription in hypoxic cells, induces expression of both CXCL12 and CXCR4 in ischemic areas $[3,4]$. Physiologically, the CXCR4CXCL12 axis has important roles in hematopoiesis, development and organization of the immune system and (ischemic) tissue repair and regeneration. In cancer, this axis has been reported to play critical roles in tumor progression, including promotion of tumor cell proliferation and survival [5], metastatic processes [6] and angiogenesis [7]. Currently, after having demonstrated anti-tumor activity in pre-clinical and animal tumor models [8], several CXCR4 antagonists are being evaluated in clinical studies for treatment of patients with hematological and solid tumors [9].

Ewing sarcoma (EWS) is an aggressive round cell sarcoma affecting bone or, rarely, soft tissue in predominantly children and young adults [10]. This tumor is characterized by specific gene fusions most commonly containing TET gene family products, and rarely other activating transcription factors [11,12]. Despite current multimodal therapies, survival of patients has not improved significantly during the past decade. Patients with refractory and/or (primary) metastatic disease have the most unfavorable prognosis, which has recently been demonstrated to be independent of gene fusion type $[13,14]$. Organs of predilection for EWS metastases are lungs and bone (marrow), which represent rich sources of CXCL12. Recently, high CXCR4 gene expression was reported to associate with metastatic phenotype in EWS [15]. Moreover, CXCL12 has been demonstrated to contribute to neovascularization and EWS tumor growth in a mouse xenograft model [16]. As yet, no information exists on CXCR4/CXCL12 protein expression and their (functional) consequences in EWS.

To gain insight into the role of the CXCR4-CXCL12 axis in EWS biology, CXCR4 expression and functionality (in the presence of CXCL12 and/or CXCR4-antagonist AMD3100) were evaluated in a large panel of therapy-naïve and metastatic tumors and cell lines, respectively. We demonstrate an expression level-dependent negative impact of CXCR4 protein expression on patients' overall survival and point to a crucial role for auto- and/or paracrine growth signaling via the CXCR4-CXCL12 axis.

\section{Methods}

\section{Ewing sarcoma patients and samples}

Formalin-fixed, paraffin-embedded therapy-naïve $(\mathrm{n}=$ $44)$ as well as (sequential) metastatic $(n=16)$ EWS samples from 47 different patients were retrieved from the Department of Pathology, LUMC and a tissue array containing 2mm-diameter tissue-cores (Institute of Pathology, Heinrich-Heine University, Dusseldorf, Germany). Histology and tumor content were verified by a specialized bone pathologist (PCWH). Diagnosis was established according to WHO criteria, including standard confirmatory immunohistochemistry and fusion transcript type. For patients with clinical information available (Additional file 1: Table S1), mean age at diagnosis was 19 years (range 1-43 years). Follow-up (mean/ median duration of follow-up: 60/44 months, respectively) provided information concerning (initial) disease extension, chemotherapy response, recurrence rate and performance state. All patient material was coded, such that code breaking and correlation with clinical data were only possible for physicians involved in treatment of the patients. Subsequent research was conducted following the ethical guidelines of the national organization of scientific societies (FEDERA).

\section{Ewing sarcoma cell lines}

EWS cell lines EW3, RD-ES, SK-ES-1, SK-N-MC, CADO-ES and STA-ET2.1 [17] and breast cancer cell line MCF-7 (ATCC, Rockville, MD) were cultured in RPMI-1640 supplemented with streptomycin/ penicillin (Invitrogen, Paisley, United Kingdom) and 10\% fetal bovine serum ((FBS); Greiner Bio-One, Alphen a/d Rijn, The Netherlands). TC71 [17] and IOR/BER (kindly provided by dr. K. Scotlandi, Instituto Orthopedico Rizzoli, Bologna, Italy) were cultured in Iscove's Modified Dulbecco's Medium supplemented with streptomycin/ penicillin and 10\% FBS. For proliferation assays, cells (at densities ranging from $3-15 \times 10^{3}$ cells/ well in 96-well-plates) were cultured for seven days in serum-free medium in the absence or presence of 100ng/ml CXCL12, 1000ng/ml AMD3100 or both. Afterwards, cell viability was measured by 3-(4,5dimethyl-thiazol-2-yl)-5-(3-carboxymethoxy-phenyl)-2(4-sulfophenyl)-2H-tetrazolium (MTS) cell viability assay (Promega Benelux, Leiden, The Netherlands), a 
colorimetric method for determining the number of viable cells in proliferation assays. The MTS tetrazolium compound is bioreduced by cells into a colored product that is soluble in tissue culture medium. The quantity of colored product as measured by the absorbance at $490 \mathrm{~nm}$ is directly proportional to the number of living cells in culture. Submission of cells to 24-hours of hypoxia was performed in $0.1 \% \mathrm{O}_{2}, 5 \% \mathrm{CO}_{2}$, balance $\mathrm{N}_{2}$ in a MiniGalaxy incubator (RS Biotech, Irvine, UK). The effectiveness of this approach for induction of hypoxia/ HIF-1 $\alpha$ expression has recently been demonstrated [18]. Cell lines were routinely screened for mycoplasma contamination. Periodical authentication was performed by Short Tandem Repeat profiling and molecular HLA typing.

\section{Antibodies and reagents}

The antibodies used for staining of antigens by immunohistochemistry and flow cytometry are described in Additional file 2: Table S2. Recombinant human CXCL12 was obtained from R\&D Systems (cat.no. 350-NS/CF; Abingdon, UK). AMD3100 (octahydrochloride hydrate) was purchased from Sigma-Aldrich (A5602; Zwijndrecht, Netherlands).

\section{Flow cytometric analysis of CXCR4 surface expression}

Flow cytometric analysis was performed on a FACScalibur (Beckton Dickinson, Franklin Lakes, NJ) and results were analyzed using Cellquest software. In short, cells were collected, centrifuged, washed in 1\% BSA/ PBS, stained with primary anti-CXCR4 antibody and, subsequently, stained with a fluorochrome-labelled secondary antibody. Ligand expression was represented as fold increase in Mean Fluorescence Intensity (MFI) over isotype control staining (MFI-ratio).

\section{Immunohistochemistry for detection of CXCR4, CXCL12 and HIF-1 expression in Ewing sarcoma tumor samples}

$4 \mu \mathrm{m}$ sections containing representative tumor, as verified by a specialized bone pathologist $(\mathrm{PCWH})$, were deparaffinized and citrate antigen retrieval was performed. Subsequent immunohistochemical stainings were performed and (semi-quantitatively) scored according to the quality control system as proposed by Ruiter et al.: the intensity of staining was scored as $0,1,2$ or 3 indicating absent, weak, moderate or strong expression, respectively. Percentages of positive cells were scored as 0 for $0 \%, 1$ for $1-5 \%, 2$ for $5-25 \%, 3$ for $25-50 \%, 4$ for $50-$ $75 \%$, and 5 for $75-100 \%$. The sum of both scores was used to identify four categories of expression: absent (02 ), weak (3-4), moderate (5-6), strong/ homogeneous (7-8) $[18,19]$. (Decalcified) tonsil tissue sections were used as positive control slides during initial optimization of immunohistochemical staining procedures. A decalcification procedure (formic acid) was applied to determine its influence on the immunoreactivity of epitopes. Quality of samples was guaranteed by (previous) immunohistochemical staining for CD99.

\section{Statistical analyses}

Statistical analyses were performed with SPSS version 16.0 software package. Survival analyses were performed according to Kaplan Meier and differences in survival curves were assessed with the log-rank test. Pearson correlation analysis was used for assessment of associations between expression levels within individual samples. T-tests or repeated measures ANOVA with Bonferroni's multiple comparison post hoc tests were used for comparison of expression levels between samples and associations between expression levels and clinicopathological parameters. $\mathrm{P}<0.05$ was considered statistically significant.

\section{Results}

CXCR4 expression in Ewing sarcoma: negative impact on survival

A panel of therapy-naïve $(n=44)$ and metastatic $(n=16)$ EWS samples were evaluated by immunohistochemistry for CXCR4 and CXCL12 expression. Variation between as well as within individual samples was observed, ranging from complete lack of expression of CXCR4 and CXCL12 to homogeneous expression of these proteins (Table 1; Figure 1A-F). Immunoreactivity for both CXCR4 and CXCL12 was exclusively localized in the cytoplasm of cells. Whereas CXCR4 was solely expressed by tumor cells, CXCL12 was observed in both tumor and stromal areas. As demonstrated in Table 1, CXCR4 expression was observed in $64 \%(28 / 44)$ of therapy-naïve and $47 \%(7 / 15)$ of metastatic tumors. CXCL12 was detectable in $65 \%(28 / 43)$ of therapy-naïve and $81 \%(13 /$ 16) of metastatic lesions. Noteworthy, stromal CXCL12 expression was detectable in nearly all cases (in 95\% (41/ 43) of therapy-naïve cases and in all metastatic lesions), regardless of CXCR4/ CXCL12 expression by tumor cells. No correlation existed between CXCR4 and CXCL12 expression levels within individual tumor samples (data not shown). Comparisons between sample types, however, demonstrated significantly higher CXCR4 expression levels in therapy-naïve as compared to (sequential) metastatic EWS cases ( $\mathrm{t}$-test, $\mathrm{p}=0.027$ ) (Table 1). Kaplan-Meier survival analysis revealed a negative impact of CXCR4 expression in therapy-naïve samples $(\mathrm{n}=30)$ on patients' overall survival (log-rank test, $\mathrm{p}=0.04$ ) (Figure 2A). Extended analyses demonstrated this impact to be expression level-dependent (log-rank test, $\mathrm{p}=0.017$ ) (Figure $2 \mathrm{~B}$ ). No such effect was observed for CXCL12 (data not shown). For cases with clinical information available $(n \geq 25)$, analysis of the 
Table 1 Immunohistochemical expression analysis of the CXCR4-CXCL12 axis in Ewing sarcoma

\begin{tabular}{|c|c|c|c|c|}
\hline \multirow[t]{2}{*}{$\mathrm{UPN}^{\mathrm{a}}$} & \multirow{2}{*}{$\begin{array}{l}\text { sample type (years } \\
\text { after diagnosis) }\end{array}$} & \multirow[t]{2}{*}{ CXCR4 ${ }^{\mathrm{b}}$} & \multicolumn{2}{|c|}{ CXCL12 } \\
\hline & & & tumor & $\overline{\text { stroma }}$ \\
\hline \multirow[t]{2}{*}{1} & lung metastasis (3) & - & - & $+/-$ \\
\hline & bone metastasis (7) & - & - & $+/-$ \\
\hline 2 & lung metastasis (2) & - & + & + \\
\hline \multirow[t]{2}{*}{3} & therapy-naive biopsy & $+/-$ & + & + \\
\hline & lung metastasis $(5,5)$ & - & - & $+/-$ \\
\hline \multirow[t]{3}{*}{4} & therapy-naive biopsy & ++ & $+/-$ & + \\
\hline & lung metastasis (3) & - & + & ++ \\
\hline & lung metastasis (4) & - & + & ++ \\
\hline \multirow[t]{2}{*}{5} & therapy-naive biopsy & ++ & $+/-$ & ++ \\
\hline & bone metastasis (1) & n.e. & $+/-$ & + \\
\hline \multirow[t]{2}{*}{6} & therapy-naive biopsy & + & ++ & n.e. \\
\hline & lung metastasis (1) & $+/-$ & $+/-$ & $+/-$ \\
\hline 7 & therapy-naive biopsy & ++ & ++ & + \\
\hline \multirow[t]{2}{*}{8} & therapy-naive biopsy & ++ & $+/-$ & + \\
\hline & bone metastasis $(2,5)$ & + & ++ & ++ \\
\hline \multirow[t]{2}{*}{9} & therapy-naive biopsy & - & + & + \\
\hline & lung metastasis $(0,5)$ & + & + & ++ \\
\hline 10 & therapy-naive biopsy & + & - & $+/-$ \\
\hline 11 & therapy-naive biopsy & - & + & $+/-$ \\
\hline 12 & therapy-naive biopsy & + & $+/-$ & + \\
\hline 13 & therapy-naive biopsy & ++ & - & $+/-$ \\
\hline 14 & therapy-naive biopsy & - & $+/-$ & + \\
\hline 15 & therapy-naive biopsy & - & - & $+/-$ \\
\hline 16 & therapy-naive biopsy & - & + & $+/-$ \\
\hline 17 & therapy-naive biopsy & - & + & + \\
\hline 18 & therapy-naive biopsy & + & $+/-$ & $+/-$ \\
\hline 19 & therapy-naive biopsy & - & $+/-$ & + \\
\hline 20 & therapy-naive biopsy & $+/-$ & - & + \\
\hline 21 & therapy-naive biopsy & - & - & $+/-$ \\
\hline 22 & therapy-naive biopsy & - & - & - \\
\hline 23 & therapy-naive biopsy & - & $+/-$ & $+/-$ \\
\hline 24 & therapy-naive biopsy & $+/-$ & $+/-$ & $+/-$ \\
\hline 25 & therapy-naive biopsy & ++ & - & - \\
\hline 26 & therapy-naive biopsy & - & + & + \\
\hline 27 & therapy-naive biopsy & + & $+/-$ & + \\
\hline 28 & therapy-naive biopsy & ++ & $+/-$ & + \\
\hline 29 & therapy naive biopsy & - & - & $+/-$ \\
\hline 30 & therapy-naive biopsy & ++ & ++ & + \\
\hline 31 & therapy-naive biopsy & + & - & ++ \\
\hline 32 & therapy-naive biopsy & + & + & + \\
\hline 33 & therapy-naive biopsy & - & - & $+/-$ \\
\hline 34 & therapy-naive biopsy & ++ & + & + \\
\hline 35 & therapy-naive biopsy & + & - & $+/-$ \\
\hline 36 & therapy-naive biopsy & + & + & + \\
\hline 37 & therapy-naive biopsy & + & - & + \\
\hline
\end{tabular}

Table 1 Immunohistochemical expression analysis of the CXCR4-CXCL12 axis in Ewing sarcoma (Continued)

\begin{tabular}{|c|c|c|c|c|}
\hline 38 & therapy-naive biopsy & ++ & n.e. & n.e. \\
\hline 39 & therapy-naive biopsy & ++ & - & $+/-$ \\
\hline 40 & therapy-naive biopsy & - & - & $+/-$ \\
\hline 41 & therapy-naive biopsy & + & + & + \\
\hline 42 & therapy-naive biopsy & + & - & $+/-$ \\
\hline 43 & therapy-naive biopsy & - & ++ & + \\
\hline 44 & therapy-naive biopsy & - & + & + \\
\hline \multirow[t]{6}{*}{45} & lung metastasis $(1,5)$ & $+/-$ & $+/-$ & ++ \\
\hline & bone metastasis $(2,5)$ & ++ & $+/-$ & + \\
\hline & lung metastasis $(2,5)$ & - & + & ++ \\
\hline & lung metastasis (4) & - & $+/-$ & ++ \\
\hline & lung metastasis (4) & $+/-$ & $+/-$ & + \\
\hline & lung metastasis (5) & + & + & + \\
\hline 46 & therapy-naive biopsy & + & + & + \\
\hline 47 & therapy-naive biopsy & $+/-$ & ++ & ++ \\
\hline
\end{tabular}

relationship with established prognostic factors in EWS [13] showed a positive correlation between CXCR4 expression in therapy-naïve samples and tumor volume at diagnosis $(<$ or $>200 \mathrm{ml}$ ) (Pearson Chi-square, $\mathrm{p}=$ 0.013). No correlations were observed with tumor site, disease extension, histologic response to chemotherapy or relapsed metastatic disease (Additional file 1: Table $\mathrm{S} 1$ ). Due to limited sample size, multivariate analysis to assess CXCR4 expression as independent prognostic factor in Ewing sarcoma could not be performed.

\section{A crucial role for the CXCR4-CXCL12 axis in promotion of Ewing sarcoma growth}

The observed correlation between CXCR4 expression and tumor volume might either be a reflection of decreased oxygen concentrations in larger/ fast-growing tumors resulting in HIF- $1 \alpha$-induced CXCR4 expression, or might be caused by increased tumor cell proliferation via auto- and/or paracrine CXCL12/ CXCR4-mediated growth signalling. To explore the first potential mechanism, hypoxia-induced CXCR4 expression and the correlation between CXCR4 and HIF-1 $\alpha$ protein expression levels were studied in EWS cell lines $(n=8)$ cultured under suboptimal conditions and therapy-naïve tumors $(\mathrm{n}=38)$, respectively.

Whereas flow cytometric analysis revealed detectable constitutive CXCR4 surface expression in all eight cell lines evaluated, only four cell lines (CADO-ES, EW3, IOR/BER, RD-ES) demonstrated substantial levels of expression of this protein (Mean Fluorescence Intensityratio (over isotype control) $>10$ ), comparable to (positive 


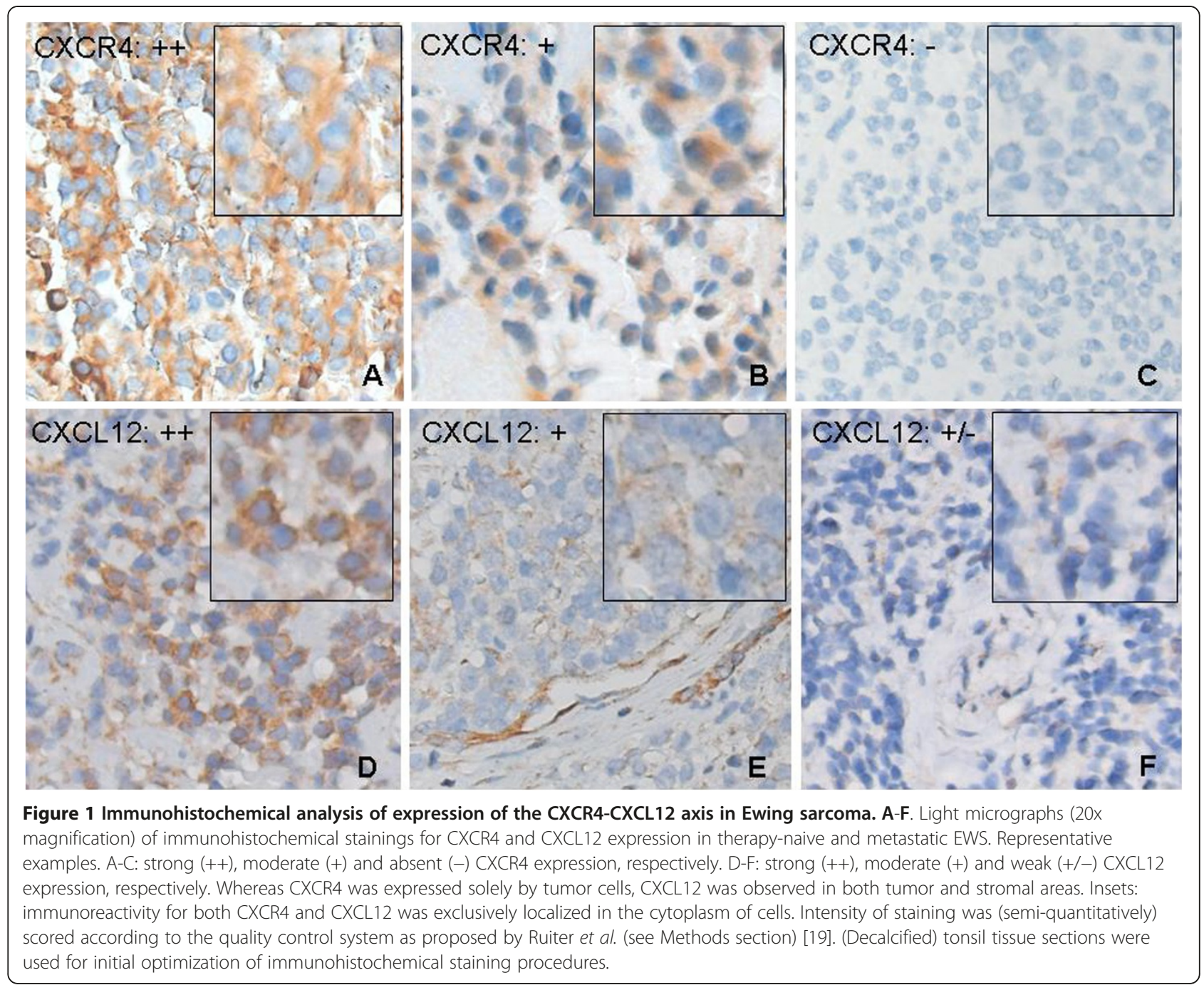

control) breast cancer cell line MCF-7 (Figure 3A). Stabilization of HIF- $1 \alpha$ protein in response to hypoxia has previously been demonstrated in EWS cell lines $[18,20]$. To evaluate the impact of hypoxia/ HIF-1 $\alpha$ activation on CXCR4 expression in EWS, cell lines expressing either substantial (CADO-ES, EW3, RD-ES) or barely detectable (STA-ET2.1, TC71) levels of CXCR4 were subjected to 24 -hours of hypoxia $\left(0.1 \% \mathrm{O}_{2}\right)$. Culture under hypoxic conditions, compared to normoxia, did not systematically affect CXCR4 surface expression (despite successful induction of VEGF, as previously described [18]) (Figure 3B). Consistent with these findings, correlation analysis of in vivo HIF-1 $\alpha$ [18] and CXCR4 expression revealed lack of correlation between expression levels of these proteins within individual tumor samples (Figure 3C).

To assess the possible contribution of the CXCR4CXCL12 axis to EWS proliferation, cell lines were cultured in serum-free medium in the absence or presence of recombinant CXCL12. As demonstrated in Figure 3D, stimulation with $100 \mathrm{ng} / \mathrm{ml}$ CXCL12 for seven days significantly increased cell numbers in cell lines expressing substantial levels of constitutive CXCR4 (CADO-ES, EW3, IOR/BER; repeated measures ANOVA with Bonferroni's multiple comparison post hoc test, $\mathrm{p}<0.001$ ), whereas no effects were observed in cell lines with minimal levels of constitutive CXCR4 expression (SK-ES-1, SK-N-MC, STA-ET2.1). Addition of CXCR4-antagonist AMD3100 at $1 \mu \mathrm{g} / \mathrm{ml}$ completely abrogated the CXCL12induced proliferation of EWS cell lines. AMD3100 treatment alone did not affect (spontaneous) cell proliferation (Figure 3D).

\section{Discussion}

Expression of the CXCR4-CXCL12 axis has been reported to coordinate events critical to tumor development and/or progression in (solid) tumors of different histological origin [2]. The present study demonstrates an (expression level-dependent) negative prognostic 


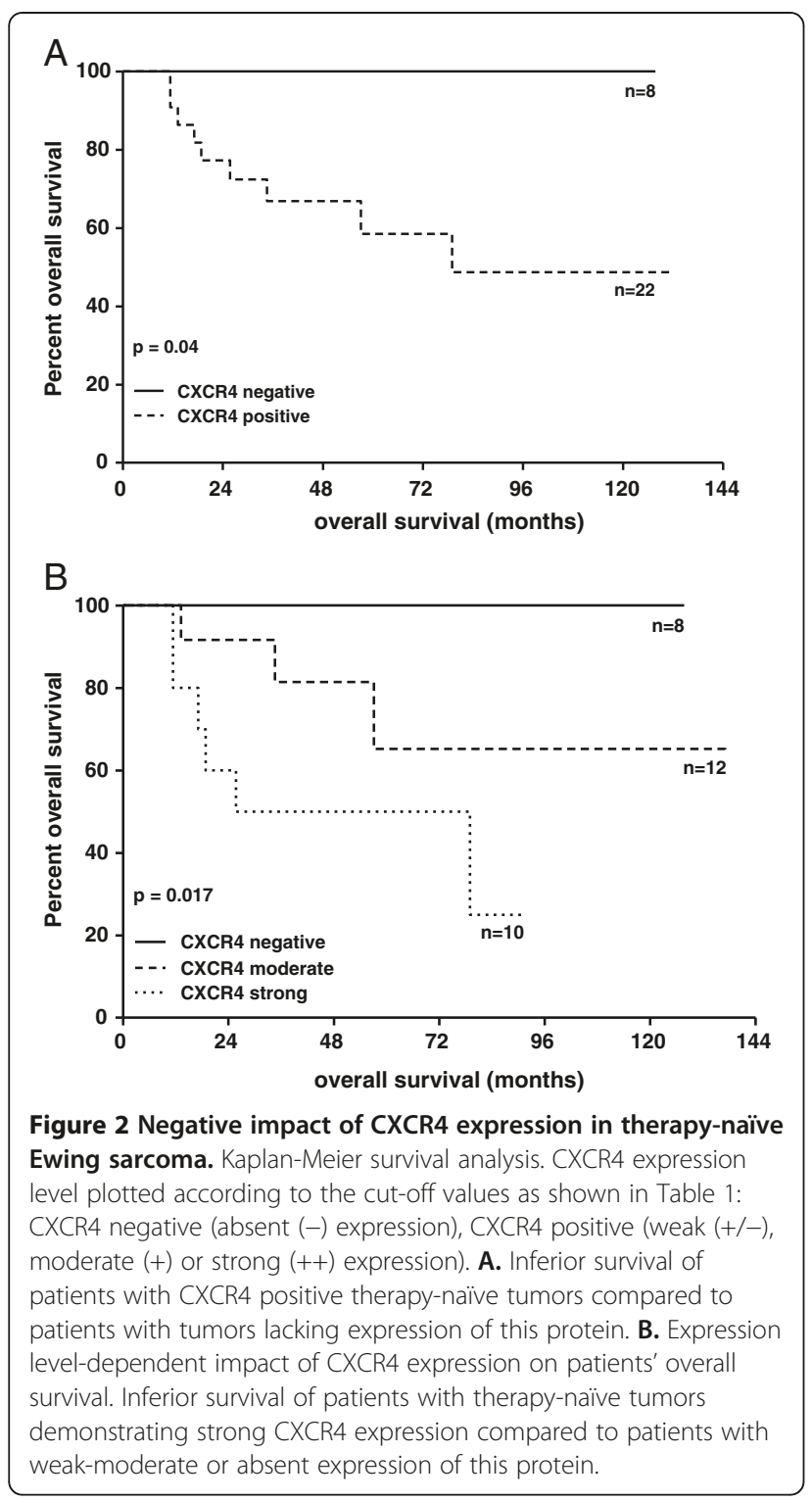

impact of CXCR4 protein expression in therapy-naïve EWS and points to a role for the CXCR4-CXCL12 axis in promotion of EWS cell growth. CXCL12-dependent modulation of tumor cell proliferation and survival (under suboptimal conditions) has been observed in several tumor types, including ovarian carcinoma [21], small cell lung cancer [22] and prostate cancer [23]. Here, we demonstrate positive correlations between CXCR4 expression levels in therapy-naïve EWS and tumor volume at diagnosis. Moreover, and consistent with previous gene expression results [15,24], we show expression of CXCL12 protein by most EWS tumors (65\%) and, explicitly, within the tumor microenvironment of virtually all (>95\%) EWS cases. Combined, these observations may reflect the existence of auto- and/ or paracrine growth stimulatory loops, mediated by the
CXCR4-CXCL12 axis. Indeed, in vitro functional analyses demonstrate CXCL12-induced proliferation of EWS cell lines expressing substantial levels of CXCR4, which could be inhibited by CXCR4-antagonist AMD3100. Addition of AMD3100 alone did not interfere with spontaneous cell proliferation, suggesting a predominant role for paracrine (stroma-derived CXCL12) rather than autocrine (tumor cell-derived CXCL12 [24]) signalling. No correlations were observed with other established prognostic factors in Ewing sarcoma. Due to limited sample size, multivariate analysis to assess CXCR4 expression as independent prognostic factor in Ewing sarcoma could not be performed. Moreover, due to the nature of this (bone) tumor, attempts to establish primary tumor cell cultures from therapy-naive biopsies for evaluation of CXCL12-induced proliferation have so far been unsuccessful. Nuclear localization of CXCR4 has been described, and demonstrated to correlate with disease progression, in several distinct cancer types $[25,26]$. Within our series of therapy-naive and metastatic Ewing sarcoma, however, no nuclear accumulation of CXCR4 has been observed.

Recently, CXCR4 gene expression was reported to associate with both EWS and osteosarcoma metastases $[15,27]$. Although we previously observed a correlation between CXCR4 gene expression and disease extension/ metastatic disease at diagnosis (unpublished results), the current study does not shown any correlation between CXCR4 protein expression and occurrence of metastatic disease. Moreover, metastatic EWS lesions demonstrated significantly lower CXCR4 protein expression levels as compared to (corresponding) therapy-naïve tumors. Reduced expression of CXCR4 in metastatic lesions as compared to corresponding primary tumors has been reported in breast carcinoma, and hypothesized to be due to CXCL12-induced internalization and degradation and/or lower microenvironmental HIF-1 $\alpha$ levels [28]. With regard to EWS, no significant differences in CXCL12 protein expression levels (in neither tumor nor stromal areas) were observed between therapy-naïve and metastatic lesions (data not shown). Moreover, although no data exist on HIF-1 $\alpha$ expression in metastatic EWS lesions, our in vitro and in vivo analyses revealed no effect of hypoxia on CXCR4 expression nor a correlation between HIF-1 $\alpha$ and CXCR4 expression levels (Figure 3B and Figure 3C, respectively). An alternative explanation for the observed reduced expression of CXCR4 in metastatic as compared to therapy-naïve EWS lesions might be that the CXCR4-CXCL12 axis is essential for retention of EWS cells within the primary tumor site, as has been described for $\mathrm{CD} 34^{+}$ hematopoietic stem cells and leukemic cells within the hematopoietic microenvironment [2] and, more recently, for osteosarcoma [29]. Hypothetically, reduced expression 


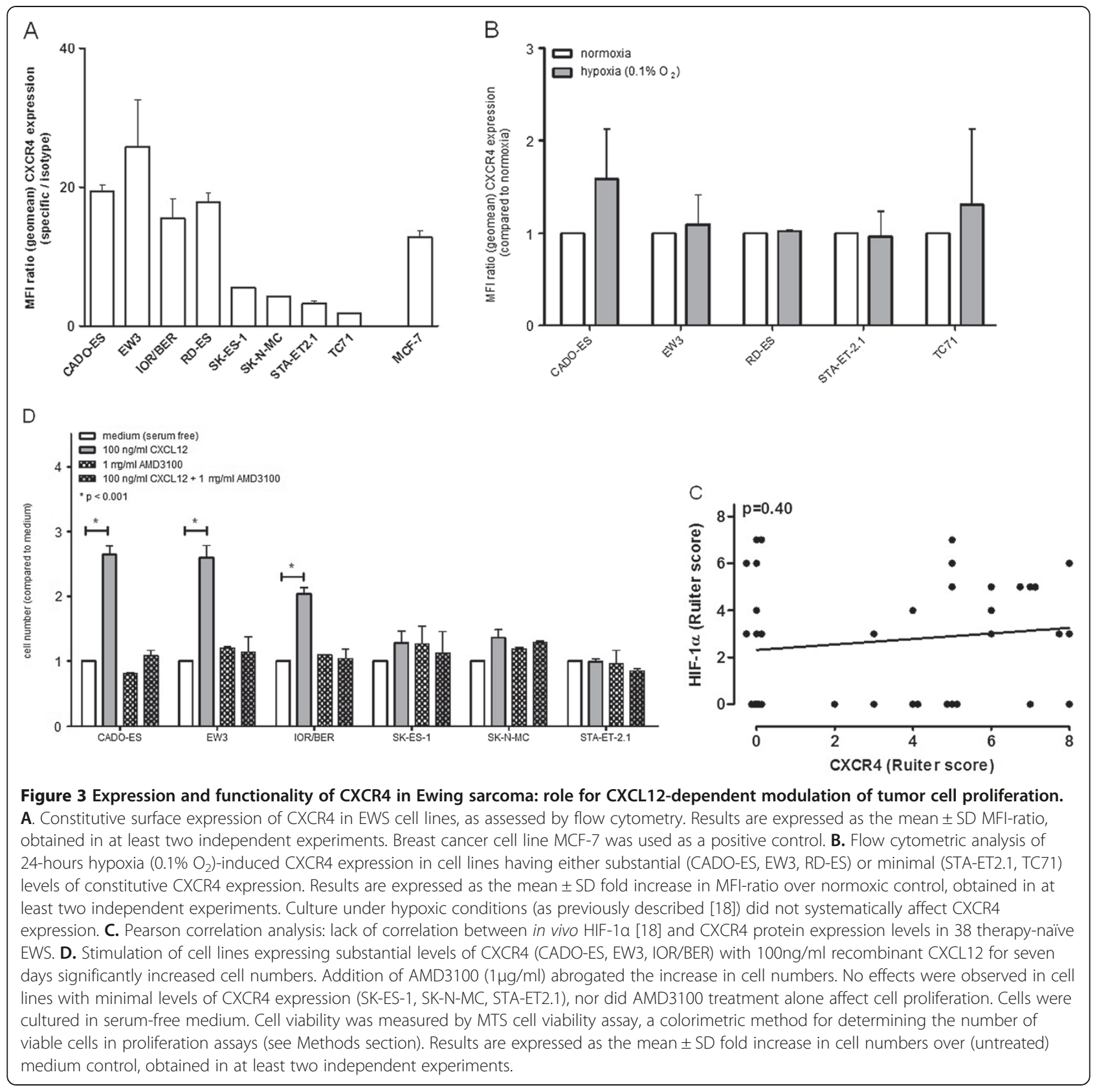

of CXCR4 might result in preferential metastasizing of individual cells, provided that alternative growth factors are present. Whether the apparent discrepancy in correlation of CXCR4 gene transcript ([15]) and CXCR4 protein expression (current study) with metastatic disease in EWS reflects true biological differences (e.g. differences at the mRNA level are not reflected at the protein level (or vice versa), due to post-transcriptional and/or -translational regulation) or are attributable to technical differences (e.g. different samples and/or sensitivity and dynamic ranges of the methods used for mRNA transcript and protein analysis) is not known. Based on our results, we delineate a role for the CXCR4-CXCL12 axis in promotion of EWS cell growth rather than its metastatic potential.

Hypoxia is a common phenomenon in (large and/or fast-growing) solid tumors, which is associated with therapy-resistance and represents an independent prognostic indicator of poor outcome. HIF- $1 \alpha$, being the best characterized inducer of gene transcription in hypoxic cells, is overexpressed in various cancer types including EWS [18,20,30], and a key role for this protein in hypoxic induction of CXCR4 has been described [3,31]. Although the observed positive correlation between 
CXCR4 expression in therapy-naïve EWS and tumor volume at diagnosis might have been indicative for hypoxia-induced HIF1 $\alpha$-dependent CXCR4 activation, our analyses did not provide support for a contribution of hypoxia to CXCR4 expression in this tumor. In addition to the observed lack of correlation between HIF- $1 \alpha$ and CXCR4 protein expression within individual tumor samples, culture of cell lines under hypoxic conditions did not affect CXCR4 surface expression. These observations are in line with results previously obtained by Aryee et al., demonstrating a lack of change in CXCR4 pathway genes upon hypoxic exposure [20].

Until rather recently, CXCR4 and CXCL12 were considered exclusive partners. However, a second CXCL12binding chemokine receptor, CXCR7, was identified and demonstrated to be involved in progression of several cancer types, including (pediatric) sarcomas [29,32,33]. In Ewing sarcoma, $C X C R 7$ gene expression was recently reported to associate with patient survival [15]. As yet, no data exist on CXCR7 protein expression in Ewing sarcoma. Our preliminary results point to limited expression of this chemokine receptor in Ewing sarcoma cell lines (CXCR7 surface expression, as assessed by flow cytometry, in $1 / 10$ cell lines). In vivo studies, using Ewing sarcoma samples or either murine or human (xenografted) tumor models may provide further insight into the role of the CXCR7-CXCR4-CXCL12 axis in Ewing sarcoma.

Hitherto, our results indicate that the CXCR4/CXCL12 axis is frequently expressed in EWS and affects tumor progression and patient survival by promoting cell growth. Successful inhibition of EWS proliferation by AMD3100, one of several CXCR4-specific antagonists that are currently being evaluated for treatment of patients with both hematological and solid tumors [9] indicates that disruption of the CXCR4-CXCL12 axis may indeed interfere with EWS progression. Integration of strategies that target CXCR4 signaling into either first- or second-line treatment regimens may represent a promising treatment option for patients with EWS.

\section{Conclusions}

Patients with Ewing sarcoma (EWS) have a poor prognosis, despite current multimodal therapy. Integration of targeted strategies into first-line treatment regimens or introduction of these approaches as second-line therapy may represent promising treatment options. Chemokine receptor CXCR4, together with its ligand CXCL12, plays critical roles in cancer progression, including growth, metastasis and angiogenesis. Lungs and bone (marrow), organs of predilection for (primary/ metastatic) EWS, represent predominant CXCL12 sources Currently, after having demonstrated anti-tumor activity in pre-clinical and animal tumor models, several CXCR4 antagonists are being evaluated in clinical studies for treatment of patients with hematological and solid tumors. Here, we demonstrate an expression level-dependent negative impact of CXCR4 protein expression on EWS patients' overall survival and provide evidence for a crucial role for the CXCR4-CXCL12 axis in promotion of EWS cell growth. Successful inhibition of EWS proliferation by CXCR4 antagonist AMD3100 indicates that disruption of the CXCR4-CXCL12 axis may indeed interfere with disease progression and provides a rationale for integration of CXCR4-targeting strategies in first- and/or second-line treatment regimens for EWS.

\section{Additional files}

Additional file 1: Table S1 (.xls). Clinical and tumor characteristics for Ewing sarcoma patients with clinical information available.

Additional file 2: Table S2 (.xIs). Antibodies used for immunohistochemistry and flow cytometry.

\section{Competing interests}

The authors declare that they have no competing interests.

\section{Authors' contributions}

All authors contributed to conception and/or design of the study. DB, SJS, SS and HJK conducted experiments and performed data analyses. DB, MWS, SS, $\mathrm{J}, \mathrm{PCWH}$ and $\mathrm{ACL}$ were involved in interpretation of data. All authors were involved in drafting and/or critical revision of the manuscript and read and approved the final manuscript.

\section{Acknowledgments}

The authors thank Dr. K. Scotlandi (Instituto Orthopedico Rizzoli, Bologna, Italy) for provision of EWS cell line IOR/BER and K.G. van der Ham (Dept. of Pathology, Leiden University Medical Center, Leiden, The Netherlands) and M.J. Ronkes for technical assistance. Financial support: European Commission (EuroBoNeT, grant no. 018814 (PCWH)); Foundation 'The Quality of Life Gala 2007' (ACL); Dutch Cancer Society (grant RUL 2003-2800 (ACL)); Federal Ministry of Education and Research Germany, BMBF (TranSaRNet) (UD); Deutsches Zentrum für Luft- und Raumfahrt e.V 01GM0869 (UD); Deutsche Krebshilfe 108128 (UD).

\section{Author details}

${ }^{1}$ Department of Pathology, Leiden University Medical Center, Leiden, The Netherlands. ${ }^{2}$ Department of Pediatrics, Leiden University Medical Center, Albinusdreef 2, 2300, RC Leiden, the Netherlands. 'Dept. of Pathology, Haartman Institute and HUSLAB, University of Helsinki and Helsinki University Central Hospital, Helsinki, Finland. ${ }^{4}$ Botnar Research Center, Nuffield Orthopedic Center, University of Oxford, Oxford, United Kingdom. ${ }^{5}$ Dept. of Pediatric Hematology and Oncology, University Hospital Muenster, Münster, Germany. ${ }^{6}$ Institute of Pathology, Heinrich-Heine University, Düsseldorf, Germany.

Received: 16 October 2012 Accepted: 24 November 2012 Published: 18 December 2012

\section{References}

1. Balkwill F: Cancer and the chemokine network. Nat Rev Cancer 2004, 4:540-550.

2. Burger JA, Kipps TJ: CXCR4: a key receptor in the crosstalk between tumor cells and their microenvironment. Blood 2006, 107:1761-1767.

3. Schioppa T, Uranchimeg B, Saccani A, Biswas SK, Doni A, Rapisarda A, Bernasconi S, Saccani S, Nebuloni M, Vago L, Mantovani A, Melillo G, Sica A: Regulation of the chemokine receptor CXCR4 by hypoxia. J Exp Med 2003, 198:1391-1402. 
4. Ceradini DJ, Kulkarni AR, Callaghan MJ, Tepper OM, Bastidas N, Kleinman ME, Capla JM, Galiano RD, Levine JP, Gurtner GC: Progenitor cell trafficking is regulated by hypoxic gradients through HIF-1 induction of SDF-1. Nat Med 2004, 10:858-864.

5. Marchesi F, Monti P, Leone BE, Zerbi A, Vecchi A, Piemonti L, Mantovani A, Allavena P: Increased survival, proliferation, and migration in metastatic human pancreatic tumor cells expressing functional CXCR4. Cancer Res 2004, 64:8420-8427.

6. Muller A, Homey B, Soto H, Ge N, Catron D, Buchanan ME, McClanahan T, Murphy E, Yuan W, Wagner SN, Barrera JL, Mohar A, Verastegui E, Zlotnik A: Involvement of chemokine receptors in breast cancer metastasis. Nature 2001, 410:50-56.

7. Orimo A, Gupta PB, Sgroi DC, Arenzana-Seisdedos F, Delaunay T, Naeem R, Carey VJ, Richardson AL, Weinberg RA: Stromal fibroblasts present in invasive human breast carcinomas promote tumor growth and angiogenesis through elevated SDF-1/CXCL12 secretion. Cell 2005, 121:335-348.

8. Duda DG, Kozin SV, Kirkpatrick ND, Xu L, Fukumura D, Jain RK: CXCL12 (SDF1-a) - CXCR4/CXCR7 pathway inhibition: an emerging sensitizer for anticancer therapies? Clin Cancer Res 2011, 17:2074-2080.

9. Hotte SJ, Hirte HW, Moretto P, lacobucci A, Wong D, Korz W, Miller WH: Final results of a Phase I/II study of CTCE-9908, a novel anticancer agent that inhibits CXCR4, in patients with advanced solid cancers. Eur J Cancer Supp/ 2008, 6:127.

10. Ushigome S, Machinami R, Sorensen PH: Ewing Sarcoma / Primitive Neuroectodermal Tumour. In World Health Organization Classification of Tumours. Pathology and Genetics of Tumours of Soft tissue and Bone. Edited by Fletcher CDM, Unni KK, Mertens F. Lyon: IARC Press; 2002:298-300.

11. Riggi N, Cironi L, Suva ML, Stamenkovic I: Sarcomas: genetics, signalling, and cellular origins. Part 1: The fellowship of TET. J Patho/ 2007, 213:4-20.

12. Szuhai $K$, ljszenga M, de Jong D, Karseladze A, Tanke HJ, Hogendoorn PC The NFATC2 gene is involved in a novel cloned translocation in a Ewing sarcoma variant that couples its function in immunology to oncology. Clin Cancer Res 2009, 15:2259-2268.

13. Le Deley MC, Delattre O, Schaefer KL, Burchill SA, Koehler G, Hogendoorn PC, Lion T, Poremba C, Marandet J, Ballet S, Pierron G, Brownhill SC, Nesslbock M, Ranft A, Dirksen U, Oberlin O, Lewis IJ, Craft AW, Jurgens H, Kovar H: Impact of EWS-ETS fusion type on disease progression in Ewing's sarcoma/peripheral primitive neuroectodermal tumor: prospective results from the cooperative Euro-E.W.I.N.G. 99 trial. J Clin Oncol 2010, 28:1982-1988.

14. van Doorninck JA, Ji L, Schaub B, Shimada H, Wing MR, Krailo MD, Lessnick SL, Marina N, Triche TJ, Sposto R, Womer RB, Lawlor ER: Current treatment protocols have eliminated the prognostic advantage of type 1 fusions in Ewing sarcoma: a report from the Children's Oncology Group. J Clin Oncol 2010, 28:1989-1994.

15. Bennani-Baiti IM, Cooper A, Lawlor ER, Kauer M, Ban J, Aryee DN, Kovar H: Intercohort gene expression co-analysis reveals chemokine receptors as prognostic indicators in Ewing's sarcoma. Clin Cancer Res 2010, 16:3769-3778

16. Reddy K, Zhou Z, Jia SF, Lee TH, Morales-Arias J, Cao Y, Kleinerman ES: Stromal cell-derived factor-1 stimulates vasculogenesis and enhances Ewing's sarcoma tumor growth in the absence of vascular endothelial growth factor. Int J Cancer 2008, 123:831-837.

17. Ottaviano L, Schaefer KL, Gajewski M, Huckenbeck W, Baldus S, Rogel U, Mackintosh C, de Alava E, Myklebost O, Kresse SH, Meza-Zepeda LA, Serra M, Cleton-Jansen AM, Hogendoorn PC, Buerger H, Aigner T, Gabbert HE, Poremba C: Molecular characterization of commonly used cell lines for bone tumor research: a trans-European EuroBoNet effort. Genes Chromosomes Cancer 2010, 49:40-51.

18. Knowles HJ, Schaefer KL, Dirksen U, Athanasou NA: Hypoxia and hypoglycaemia in Ewing's sarcoma and osteosarcoma: regulation and phenotypic effects of hypoxia-inducible factor. BMC Cancer 2010, 10:372-381

19. de Hooge ASK, Berghuis D, Santos SJ, Mooiman E, Romeo S, Kummer JA, Egeler RM, van Tol MJ, Melief CJ, Hogendoorn PCW, Lankester AC: Expression of cellular FLICE inhibitory protein, caspase-8, and protease inhibitor-9 in Ewing sarcoma and implications for susceptibility to cytotoxic pathways. Clin Cancer Res 2007, 13:206-214.

20. Aryee DN, Niedan S, Kauer M, Schwentner R, Bennani-Baiti IM, Ban J, Muehlbacher K, Kreppel M, Walker RL, Meltzer P, Poremba C, Kofler R, Kovar
$\mathrm{H}$ : Hypoxia modulates EWS-FLI1 transcriptional signature and enhances the malignant properties of Ewing's sarcoma cells in vitro. Cancer Res 2010, 70:4015-4023.

21. Scotton CJ, Wilson JL, Scott K, Stamp G, Wilbanks GD, Fricker S, Bridger G, Balkwill FR: Multiple actions of the chemokine CXCL12 on epithelial tumor cells in human ovarian cancer. Cancer Res 2002, 62:5930-5938.

22. Kijima T, Maulik G, Ma PC, Tibaldi EV, Turner RE, Rollins B, Sattler M, Johnson $\mathrm{BE}$, Salgia R: Regulation of cellular proliferation, cytoskeletal function, and signal transduction through CXCR4 and c-Kit in small cell lung cancer cells. Cancer Res 2002, 62:6304-6311.

23. Sun YX, Wang J, Shelburne CE, Lopatin DE, Chinnaiyan AM, Rubin MA Pienta KJ, Taichman RS: Expression of CXCR4 and CXCL12 (SDF-1) in human prostate cancers (PCa) in vivo. J Cell Biochem 2003, 89:462-473.

24. Berghuis D, Santos SJ, Baelde HJ, Taminiau AH, Egeler RM, Schilham MW, Hogendoorn PC, Lankester AC: Pro-inflammatory chemokine-chemokine receptor interactions within the Ewing sarcoma microenvironment determine CD8(+) T-lymphocyte infiltration and affect tumour progression. J Pathol 2011, 223:347-357.

25. Wang L, Wang Z, Yang B, Yang Q, Wang L, Sun Y: CXCR4 nuclear localization follows binding of its ligand SDF-1 and occurs in metastatic but not primary renal cell carcinoma. Oncol Rep 2009, 22:1333-1339.

26. Wang SC, Lin JK, Wang HS, Yang SH, Li AF, Chang SC: Nuclear expression of CXCR4 is associated with advanced colorectal cancer. Int J Colorectal Dis 2010, 25:1185-1191.

27. Namlos HM, Kresse SH, Muller CR, Henriksen J, Holdhus R, Saeter G, Bruland OS, Bjerkehagen B, Steen VM, Myklebost O: Global gene expression profiling of human osteosarcomas reveals metastasisassociated chemokine pattern. Sarcoma 2012, 2012:639038.

28. Shim H, Lau SK, Devi S, Yoon Y, Cho HT, Liang Z: Lower expression of CXCR4 in lymph node metastases than in primary breast cancers: potential regulation by ligand-dependent degradation and HIF-1alpha. Biochem Biophys Res Commun 2006, 346:252-258.

29. Baumhoer D, Smida J, Zillmer S, Rosemann M, Atkinson MJ, Nelson PJ, Jundt G, von Luettichau I, Nathrath M: Strong expression of CXCL12 is associated with a favorable outcome in osteosarcoma. Mod Pathol 2012 25:522-528.

30. van der Schaft DW, Hillen F, Pauwels P, Kirschmann DA, Castermans K Egbrink MG, Tran MG, Sciot R, Hauben E, Hogendoorn PC, Delattre O, Maxwell PH, Hendrix MJ, Griffioen AW: Tumor cell plasticity in Ewing sarcoma, an alternative circulatory system stimulated by hypoxia. Cancer Res 2005, 65:11520-11528.

31. Staller P, Sulitkova J, Lisztwan J, Moch H, Oakeley EJ, Krek W: Chemokine receptor CXCR4 downregulated by von Hippel-Lindau tumour suppressor pVHL. Nature 2003, 425:307-311.

32. Burns JM, Summers BC, Wang Y, Melikian A, Berahovich R, Miao Z, Penfold ME, Sunshine MJ, Littman DR, Kuo CJ, Wei K, McMaster BE, Wright K, Howard MC, Schall TJ: A novel chemokine receptor for SDF-1 and I-TAC involved in cell survival, cell adhesion, and tumor development. J Exp Med 2006, 203:2201-2213.

33. Grymula K, Tarnowski M, Wysoczynski M, Drukala J, Barr FG, Ratajczak J, Kucia M, Ratajczak MZ: Overlapping and distinct role of CXCR7-SDF-1/ ITAC and CXCR4-SDF-1 axes in regulating metastatic behavior of human rhabdomyosarcomas. Int J Cancer 2010, 127:2554-2568.

doi:10.1186/2045-3329-2-24

Cite this article as: Berghuis et al:: The CXCR4-CXCL12 axis in Ewing sarcoma: promotion of tumor growth rather than metastatic disease. Clinical Sarcoma Research 2012 2:24. 